\author{
Marquette University \\ e-Publications@Marquette
}

$5-21-2015$

\title{
Mixed Quantum/Classical Approach for Description of Molecular Collisions in Astrophysical Environments
}

Alexander Semenov

Marquette University

Dmitri Babikov

Marquette University, dmitri.babikov@marquette.edu

Follow this and additional works at: https://epublications.marquette.edu/chem_fac

Part of the Chemistry Commons

\section{Recommended Citation}

Semenov, Alexander and Babikov, Dmitri, "Mixed Quantum/Classical Approach for Description of Molecular Collisions in Astrophysical Environments" (2015). Chemistry Faculty Research and Publications. 474.

https://epublications.marquette.edu/chem_fac/474 


\title{
Mixed Quantum/Classical Approach for Description of Molecular Collisions in Astrophysical Environments
}

\author{
Alexander Semenov \\ Chemistry Department, Marquette University, \\ Milwaukee, WI \\ Dmitri Babikov \\ Chemistry Department, Marquette University, \\ Milwaukee, WI
}




\section{Abstract}

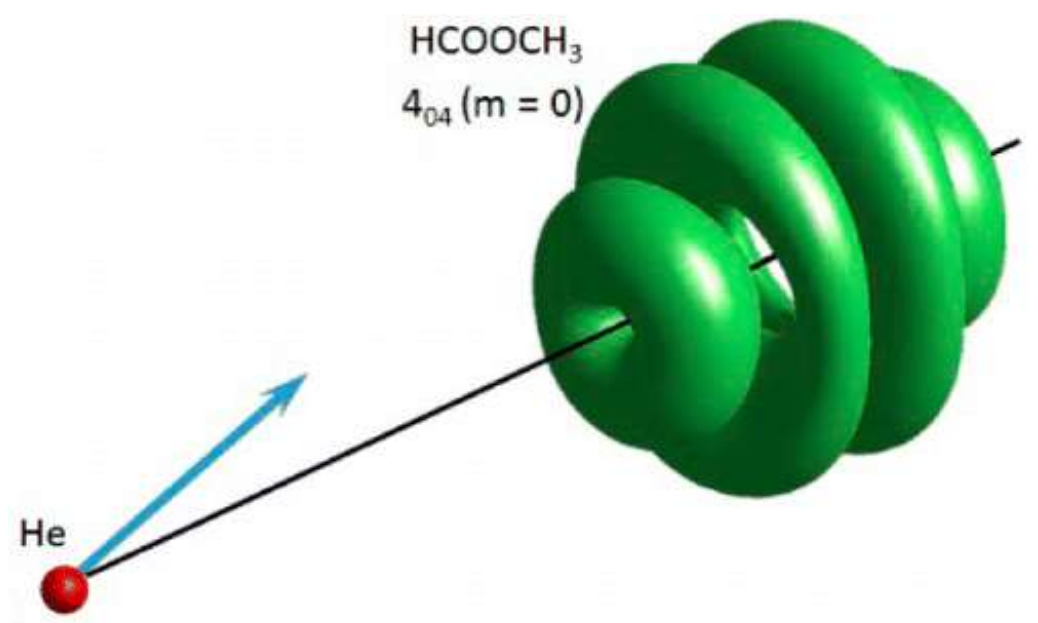

An efficient and accurate mixed quantum/classical theory approach for computational treatment of inelastic scattering is extended to describe collision of an atom with a general asymmetric-top rotor polyatomic molecule. Quantum mechanics, employed to describe transitions between the internal states of the molecule, and classical mechanics, employed for description of scattering of the atom, are used in a self-consistent manner. Such calculations for rotational excitation of $\mathrm{HCOOCH}_{3}$ in collisions with He produce accurate results at scattering energies above $15 \mathrm{~cm}^{-1}$, although resonances near threshold, below $5 \mathrm{~cm}^{-1}$, cannot be reproduced. Importantly, the method remains computationally affordable at high scattering energies (here up to $1000 \mathrm{~cm}^{-1}$ ), which enables calculations for larger molecules and at higher collision energies than was possible previously with the standard full-quantum approach. Theoretical prediction of inelastic cross sections for a number of complex organic molecules observed in space becomes feasible using this new computational tool.

Keywords: collisional energy transfer; computational methods; inelastic scattering; quantum dynamics

To the present day more than 200 molecular species have been detected in space, including some complex organic molecules and long carbon chains. ${ }^{1-6}$ For quantitative interpretation of their observed spectra the inelastic state-to-state transition cross sections for these molecules collided with background gases $\left(\mathrm{H}_{2}\right.$ and $\mathrm{He}$ in the interstellar medium, or $\mathrm{H}_{2} \mathrm{O}$ in cometary environment) are needed, often in a broad range of collision energies. This information, however, is largely missing, and there is no simple way of determining it from experiments. Collisional cross sections could be computed within 
quantum-mechanical framework ${ }^{7,8}$ by numerically solving the Schrödinger equation for nuclear motion of colliding partners, but such brute-force calculations are computationally affordable for only some of the smallest molecules and only at low energies of collision. ${ }^{1,9}$ For example, quantum calculations of $\mathrm{H}_{2} \mathrm{O}+\mathrm{H}_{2}$ in the required range of collision energies (up to $T=1500 \mathrm{~K}$ ) have been achieved just recently ${ }^{10}$ and represent the state-of-the-art in the field. Calculations for $\mathrm{HCOOCH}_{3}+\mathrm{He}$ have been attempted ${ }^{11}$ but were shown to be computationally affordable only for collision energies below $30 \mathrm{~cm}^{-1}$. Such important process as scattering of $\mathrm{H}_{2} \mathrm{O}+\mathrm{H}_{2} \mathrm{O}$ in the desired energy range (up to $T \approx 300 \mathrm{~K}$ ) is also beyond the reach of theorists. This is quite demonstrative because many astrophysically important molecules are larger than $\mathrm{H}_{2} \mathrm{O}$, for example: $\mathrm{CH}_{3} \mathrm{CHO}, \mathrm{CH}_{3} \mathrm{OCH}_{3}$, $\mathrm{C}_{2} \mathrm{H}_{5} \mathrm{CN}, \mathrm{C}_{6} \mathrm{H}_{2}, \mathrm{CH}_{3} \mathrm{C}_{4} \mathrm{H}$, and $\mathrm{HC}_{5} \mathrm{~N} .{ }^{1,11-13}$ One should admit that although a significant progress has been made on inelastic scattering calculations for small molecules and at low collision energies, ${ }^{1,2}$ the standard full-quantum approach is basically stuck when the molecules are heavy and the collision energy is large, when molecule-molecule collisions are important, or when in addition to rotation the vibrational motion (torsion, bending) has to be taken into account. Thus, it is desirable to develop an alternative or complementary approach that would allow circumventing the computational difficulties by employing some kind of approximation.

Recently we developed a mixed quantum/classical theory (MQCT) for inelastic scattering ${ }^{14,15}$ where the internal motion of the molecule is still treated quantum mechanically, while the scattering of the atom is described approximately using classical mechanics (Newtonian trajectories), which reduces the computational cost dramatically. We rigorously tested this theory by applying it to $\mathrm{CO}+$ $\mathrm{He},{ }^{16} \mathrm{H}_{2}+\mathrm{He}^{17} \mathrm{~N}_{2}+\mathrm{Na},{ }^{18}$ and $\mathrm{H}_{2} \mathrm{O}+\mathrm{He},{ }^{19}$ and obtained very good agreement with the standard full-quantum treatment in a broad range of collision energies (computationally affordable for these simpler molecules). We found that MQCT gives a detailed description of the scattering process, even at the level of differential cross sections in the forward scattering quantum regime, ${ }^{17,18}$ which is a known unresolved issue for all semiclassical methods. ${ }^{20}$ At higher collision energies it reproduces the full-quantum results almost exactly, but even at low collision energies the predictions of MQCT are reasonable. ${ }^{16-19}$ The only feature that MQCT does not reproduce is scattering resonances at very

Journal of Physical Chemistry Letters, Vol 6, No. 10 (May 21, 2015): pg. 1854-1858. DOI. This article is (C) American Chemical Society and permission has been granted for this version to appear in e-Publications@Marquette. American Chemical Society does not grant permission for this article to be further copied/distributed or hosted elsewhere without the express permission from American Chemical Society. 
low collision energies, near the threshold of the excitation process. In the past decade, a family of closely related theoretical methods has been developed using the quantized Hamiltonian dynamics (QHD) approach. ${ }^{21-23}$ They also utilize the mixed quantum/classical ideas based on the Ehrenfest mean-field potential but in a different fashion.

It is interesting that foundations of this theory were laid out by Billing in the 1980 s and $90 s,{ }^{24,25}$ but then it was largely abandoned without been properly tested. Our recent progress on diatomics and triatomics was rapid, ${ }^{14-19}$ which demonstrated that MQCT can be used as an efficient predictive computational tool for small molecules; however, it is often a challenge to extend an approximate method onto the complex systems. New problems may appear, such as difficulty of accurate representation of the potential energy surface, density of rotational spectrum of a heavy asymmetric-top rotor, and higher costs of numerical calculations, to name just a few. Our goal nowadays is to apply MQCT to several complicated problems, such as small organic molecules of astrophysical importance, which would represents a major step forward.

We report MQCT results for rotational excitation of $\mathrm{HCOOCH}_{3}$ (methyl formate) by He in the range of collision energies up to 1000 $\mathrm{cm}^{-1}$, typical for warm star-forming regions. To our best knowledge this is the largest molecule ever considered for the inelastic scattering calculations. ${ }^{1}$ The full-quantum scattering results (available for this process at low energy only ${ }^{11}$ ) serve as a benchmark and demonstrate that our approach is accurate. Our general conclusion is that at medium and higher collision energies, when quantum scattering resonances are not important, MQCT can confidently replace the fullquantum scattering approach. MQCT remains computationally affordable and enables theoretical predictions of inelastic cross sections for larger molecules and at higher collision energies than was possible before, in particular, for the processes of astrochemical importance.

The details of our theory are given in the Supporting Information. In a nutshell, we propagate batches of MQCT trajectories to sample the classical impact parameter for collisions between He and $\mathrm{HCOOCH}_{3}$. Such trajectories are driven by the mean-field potential, averaged over the rotational wave function of the molecule (see the

Journal of Physical Chemistry Letters, Vol 6, No. 10 (May 21, 2015): pg. 1854-1858. DOI. This article is (C) American Chemical Society and permission has been granted for this version to appear in e-Publications@Marquette. American Chemical Society does not grant permission for this article to be further copied/distributed or hosted elsewhere without the express permission from American Chemical Society. 
TOC image). Typically, the number of trajectories on order of a hundred is sufficient to capture the dependence of transition probability on the impact parameter, as shown in Figure 1. As trajectory progresses, the evolution of rotational wave function of the molecule is determined by the system of coupled differential equations that includes elements of the transition matrix due to interaction with the atom. In this way, the scattering motion of the atom and the rotational motion of the molecule affect each other, and the quantum and classical degrees of freedom are treated self-consistently. The energy is exchanged between translation and rotation, while the total energy is conserved. Figure 2 illustrates evolution of state populations along a typical MQCT trajectory. In this picture the atom-molecule encounter occurs in the short time interval between $t \approx 70$ and $100 \times$ $10^{3}$ a.u. Two scenarios of rotational excitations can be identified. For the majority of states the population starts growing exponentially on the precollisional stage and remains almost constant on the post collisional stage. Examples are $202,212,404,1_{11}$, and $1_{01}$. However, for some states the population starts growing much later, basically during the collision, and continues evolving at the postcollisional stage. Examples are $2_{11}$ and $1_{10}$. This difference comes from the fact that the first group of states is populated by transitions directly from the ground state $0_{00}$ due to potential coupling. The second group of states is populated due to centrifugal coupling with other excited states, indirectly, and only after those intermediate states receive enough population. This leads to a later start, longer time evolution, and lower transition probabilities (see Figure 2). As discussed later, such transitions are often neglected by the coupled-states (or centrifugally sudden) approximation, but they are included in MQCT calculations. In either case, the populations of rotational states of the molecule at the end of trajectories are used to compute the state-to-state transition cross sections. ${ }^{16}$ 
NOT THE PUBLISHED VERSION; this is the author's final, peer-reviewed manuscript. The published version may be accessed by following the link in the citation at the bottom of the page.

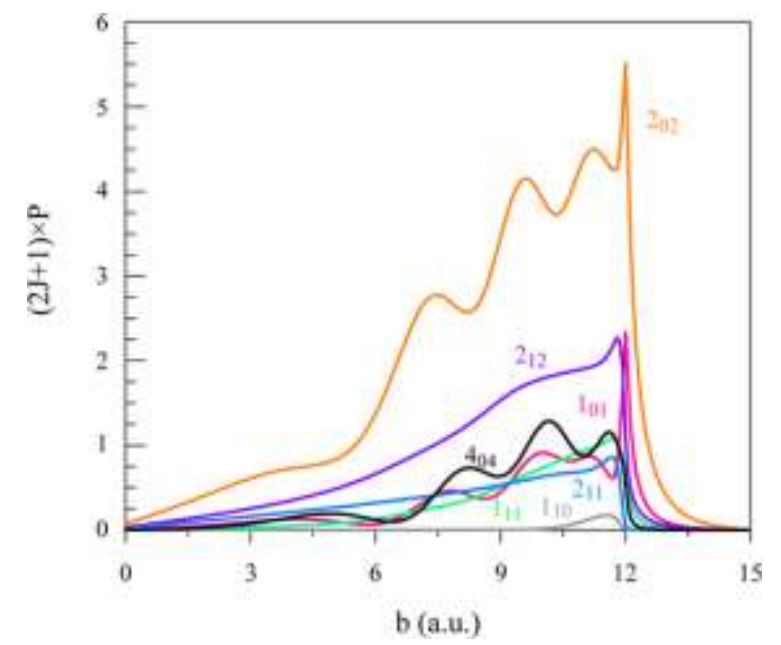

Figure 1. Dependence of quantum transition probability on classical impact parameter for excitation (by He collision) of $\mathrm{HCOOCH}_{3}$ from its ground rotational state $0_{0,0}$ into several final rotational states. The collision energy is $17 \mathrm{~cm}^{-1}$. The color of state labels corresponds to the color of curves.

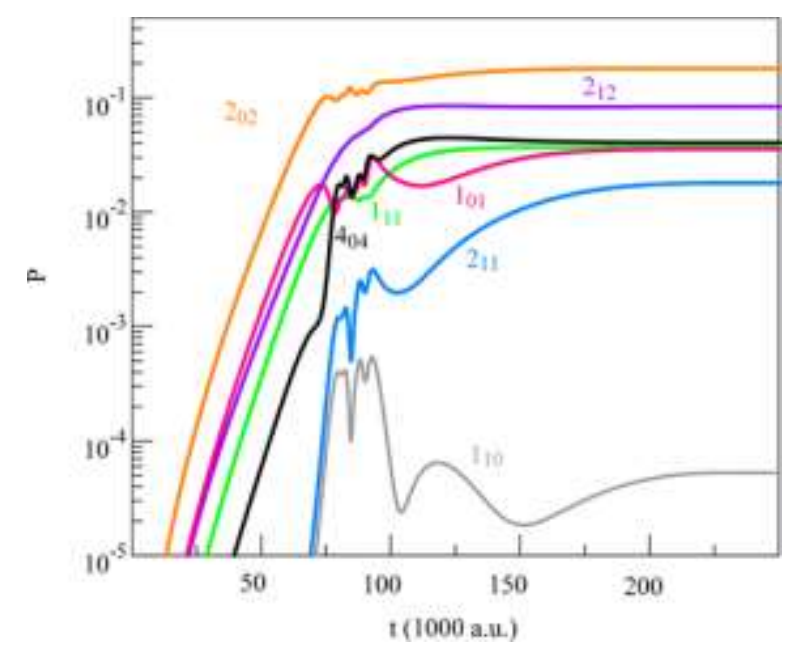

Figure 2. Evolution of state populations in $\mathrm{HCOOCH}_{3}$ along a typical MQCT trajectory that describes its collision with He. The collision energy is $17 \mathrm{~cm}^{-1}$, and the impact parameter is $10.5 a_{0}$. The color of state labels corresponds to the color of curves.

In Figure 3 we compare our MQCT results against the available full-quantum results from ref 11 for rotational excitation of the ground state $0_{00}$ of $\mathrm{HCOOCH}_{3}$ to several low-lying rotationally excited states using the same potential energy surface. For the most important states (large cross sections) the agreement is excellent: In the energy range $15-30 \mathrm{~cm}^{-1}$, where quantum resonances level off, the typical differences are on the order of only 5\%. For the less important state $1_{1,0}$ (small cross section) the difference is somewhat larger near, but it should be stressed that our MQCT results are fully converged with

Journal of Physical Chemistry Letters, Vol 6, No. 10 (May 21, 2015): pg. 1854-1858. DOI. This article is (C) American Chemical Society and permission has been granted for this version to appear in e-Publications@Marquette. American Chemical Society does not grant permission for this article to be further copied/distributed or hosted elsewhere without the express permission from American Chemical Society. 
respect to the number of partial waves $\left(J_{\max }=20\right)$, while convergence of the full-quantum data from ref 11 for the state $1_{1,0}$ was reported as $\sim 20 \%$ (i.e., not entirely converged, due to high computational cost of better calculations), which explains larger difference obtained for this state. To make comparison meaningful, the rotational basis set size in our MQCT calculations was taken the same as in ref 11 , namely, $j_{\max }=$ 14 (225 channels).

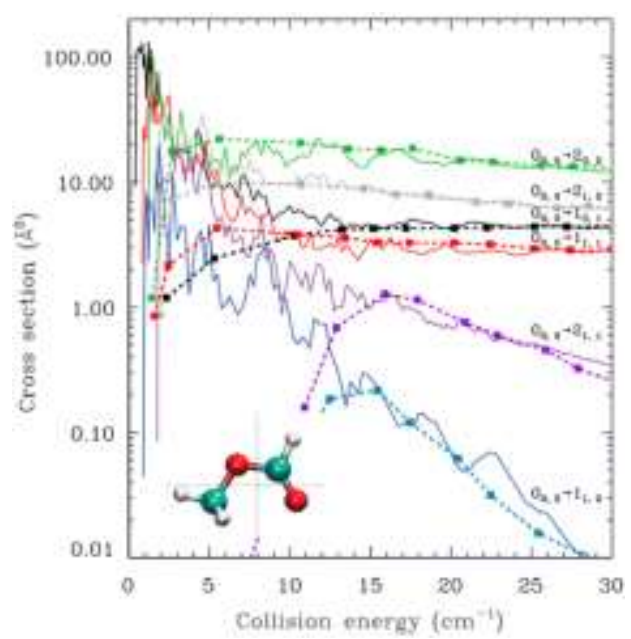

Figure 3. Inelastic cross sections for excitation of several rotationally excited states of $\mathrm{HCOOCH}_{3}$ from its ground state in collisions with He at low scattering energies. Results of the full-quantum calculations from ref 11 are shown by solid lines. Our MQCT results are shown by symbols of the same color (connected by dashed lines for clarity). The inset shows the molecular structure of methyl formate. Reproduced with permission from ref 11 . Copyright 2011 AIP Publishing LLC.

In the lower energy range, $5-15 \mathrm{~cm}^{-1}$ in Figure 3 , the results of MQCT for the most important transitions are still reasonable. The accuracy of MQCT drops significantly only at collision energies below 5 $\mathrm{cm}^{-1}$, where quantum resonances dominate. At these low collision energies many trajectories describe orbiting of the He atom around the molecule, which is classical analogue of quantum resonance. A good recipe for analysis of such trajectories is yet to be found. For now, we simply removed them from consideration, focusing on nonresonant contribution to the process.

Overall, Figure 3 demonstrates that at collision energies near 30 $\mathrm{cm}^{-1}$ the MQCT method gives an accurate description of the inelastic $\mathrm{HCOOCH}_{3}+\mathrm{He}$ collisions. Our prior experience with MQCT applied to four different systems in a broad energy range ${ }^{16-19}$ shows that its accuracy always improves as collision energy is raised. So, based on

Journal of Physical Chemistry Letters, Vol 6, No. 10 (May 21, 2015): pg. 1854-1858. DOI. This article is (C) American Chemical Society and permission has been granted for this version to appear in e-Publications@Marquette. American Chemical Society does not grant permission for this article to be further copied/distributed or hosted elsewhere without the express permission from American Chemical Society. 
the results of Figure 3, we can expect that at energies above $30 \mathrm{~cm}^{-1}$ (where there are no quantum data available) our MQCT predictions of the inelastic scattering cross sections would be reliable, with errors $<5 \%$. In Figure 4 we present the MQCT predictions for excitation of the 20 most important rotational states of $\mathrm{HCOOCH}_{3}$, starting from the ground state $0_{00}$, in the collisional energy range expanded by a factor of more than 30 , up to $1000 \mathrm{~cm}^{-1}$. These states are $1_{01}, 1_{11}, 2_{02}, 2_{12}$, $222,2_{21}, 3_{03}, 3_{13}, 3_{21}, 3_{31}, 4_{04}, 4_{14}, 4_{22}, 4_{40}, 5_{05}, 5_{33}, 5_{33}, 5_{41}, 6_{16}, 6_{24}$, and $77_{17}$. The dependencies in Figure 4 are rather smooth, although some of them are not entirely monotonic and the overall picture is rather complicated. The reason for this is that $\mathrm{HCOOCH}_{3}$ is a heavy rotor and the spectrum of its states is rather dense, with many stateto-state transitions accessible and participating actively in the energy transfer. In these MQCT calculations the number of rotational channels was around 1130 , with typical values of $J_{\max }$ around 120 . This is a very large number of channels. The full-quantum calculations with such number of channels would not be practical. For all calculations in this paper we used the potential energy surface from ref 11 . This surface is based on high-level ab initio electronic structure calculations (CCSD(T)/aug-cc-pVTZ), employs fitting of 476 data points by an analytic function, and is expected to be accurate up to collision energy of $1000 \mathrm{~cm}^{-1}$. Note that we do not employ expansion of the PES over the basis set of spherical harmonics. This procedure, standard for small and simple molecules, does not work well for larger and complicate molecules, ${ }^{11}$ such as methyl formate. Elements of the potential coupling matrix were computed numerically, as explained in the Supporting Information.

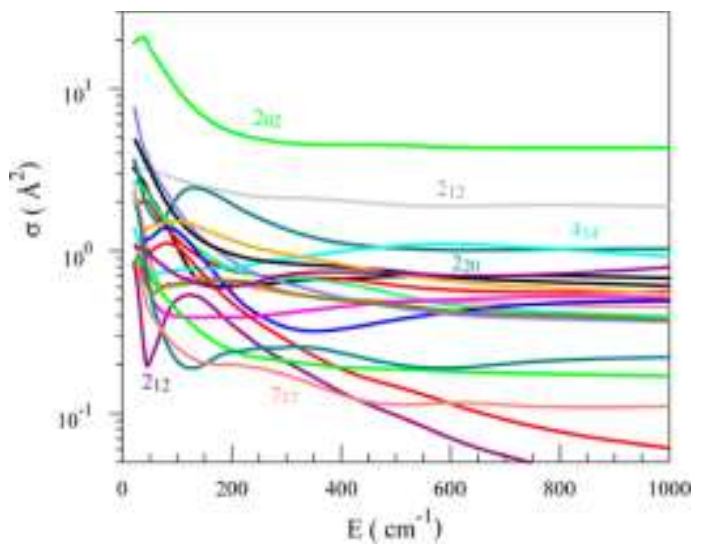

Figure 4. Inelastic cross sections for excitation of 20 most important rotationally excited states of $\mathrm{HCOOCH}_{3}$ from its ground state computed by MQCT for a broad range

Journal of Physical Chemistry Letters, Vol 6, No. 10 (May 21, 2015): pg. 1854-1858. DOI. This article is (C) American Chemical Society and permission has been granted for this version to appear in e-Publications@Marquette. American Chemical Society does not grant permission for this article to be further copied/distributed or hosted elsewhere without the express permission from American Chemical Society. 
of collision energies. Some of the final states are labeled, and others are listed in the text.

One known way to make the full-quantum scattering calculations more affordable is the coupled-states (CS) approximation, ${ }^{26}$ in which some transitions (between states with different values $\mathrm{m}$ of projection of the angular momentum $j$ ) are neglected to ease calculations. The CS method is usually employed at higher collision energies. Interestingly, within MQCT one can also formulate the CS approximation and test it by comparing its results against the fully coupled MQCT, which we will now call CC-MQCT. ${ }^{17}$ Results of such CS-MQCT calculations are presented in Figure 5. Comparison of these data against CC-MQCT (presented in Figure 3) shows that although the general behavior of energy dependence is similar, the absolute values of CS cross sections are often different (e.g., by a factor of up to $\times 1.8$ for state 220 , by a factor of up to $\times 2.1$ for state $4_{12}$, and by a factor of up to $\times 1.4$ for state 202 ). For some transitions these differences vanish as collision energy reaches 1000 $\mathrm{cm}^{-1}$, but for several other state-to-state transitions large differences survive even at higher energies. Needless to say, some transitions do not happen at all within the CS approximation (e.g., excitation of states $1_{10}$ and $2_{11}$ ). One important conclusion is that the CS approximation is not particularly accurate for the $\mathrm{HCOOCH}_{3}+\mathrm{He}$ system in the considered energy range. One should not expect that the quantum CS calculations for this molecule will be accurate. Thus, the fully coupled version of MQCT is, perhaps, the only practical way of doing accurate calculations for this and other similar organic molecules of astrophysical relevance.

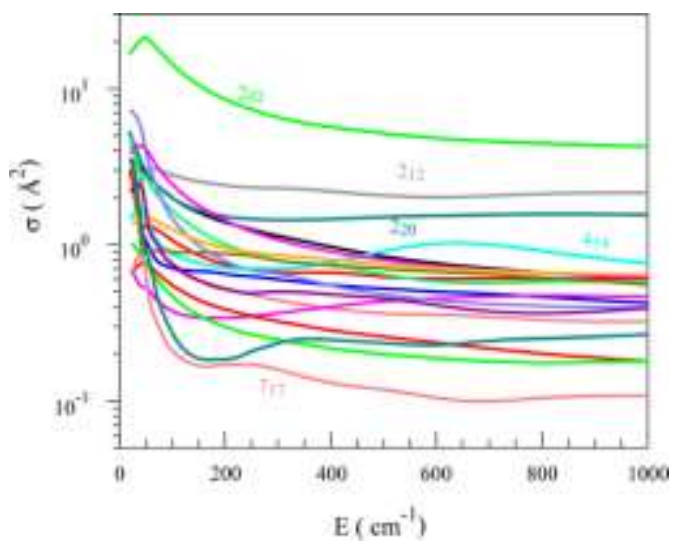

Figure 5. Same as in Figure 4 but computed using CS-approximation within MQCT framework. 
The numerical cost of our MQCT calculations is represented by Figure 6 . The CS version of MQCT is faster than the fully coupled MQCT by a factor of roughly 20 . The scaling law (computational cost vs number of included channels) is $n^{2.4}$ for the fully coupled MQCT and is $n^{2}$ for CS-MQCT. These numbers are taken directly from calculations presented in Figures 3 and 4 and represent a practical measure of the computational cost of the method in a range of collision energies. We also did run an idealized test of performance, when MQCT calculations were done at one representative collision energy, while the number of included channels was varied in a broad range. In such tests the scaling law of the fully coupled MQCT was $n^{2.5}$. For comparison, the full-quantum calculations (e.g., using Hibridon or MOLSCAT) ${ }^{27,28}$ are usually said to scale as $n^{3}$ with respect to the number of channels, but the cost of converging the quantum calculations with respect to the number of partial waves (which depends on collision energy and the reduced mass) should be added to that, leading, in practice, to the total cost on the order of $n^{5}$ or $n^{6}$. In contrast, MQCT has no such "overhead" because scattering of the atom is treated classically. Thus, the scaling properties of MQCT are more favorable than those of the full-quantum method, and the advantages are particularly significant for heavier collision partners and at higher collision energies; however, it should also be mentioned that at this point we have only undertaken some basic optimizations of our code. Development of an efficient computer program should reduce the computational costs of MQCT even further.

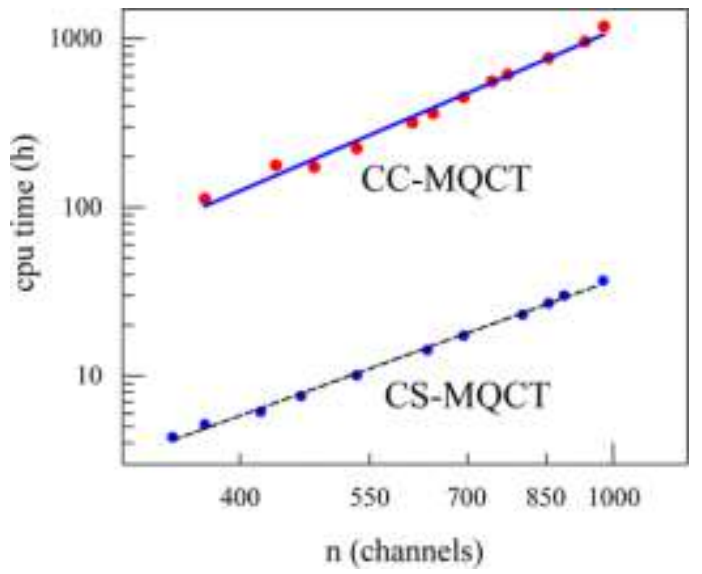

Figure 6. Numerical performance of MQCT, observed for its fully coupled CC version and for the approximate CS version. Logarithmic scale is used for both horizontal and vertical axes. Dashed lines show fits by two different power functions. 
In conclusion, we carried out the rotationally inelastic scattering calculations for collision of methyl formate with helium atom within the framework of the mixed quantum/classical theory. First, we compared our results against the full-quantum results available at low collision energies and found a very good agreement in the range between 15 and $30 \mathrm{~cm}^{-1}$. Next, we significantly expanded the range of collision energies (by a factor of more than 30 , up to $1000 \mathrm{~cm}^{-1}$ ), covering a practically useful scattering regime. Importantly, the standard fullquantum calculations are computationally unaffordable for such a large molecule in this scattering regime. One can look at MQCT as a method that is complementary to the standard full-quantum method. Namely, at low collision energies one may want to do the full-quantum scattering calculations because they are affordable and because some quantum features, like scattering resonances, may be important; however, at higher collision energies, when the full-quantum calculations become unaffordable (and, in fact, unnecessary) one may want to switch to the mixed quantum/classical theory. It is feasible to apply MQCT to a number of complicated processes of astrophysical relevance, such as collisions of complex organic molecules $\left(\mathrm{CH}_{3} \mathrm{CHO}\right.$, $\mathrm{CH}_{3} \mathrm{OCH}_{3}, \mathrm{C}_{2} \mathrm{H}_{5} \mathrm{CN}$, and $\left.\mathrm{HCOOCH}_{3}\right)$ or linear carbon chains $\left(\mathrm{C}_{6} \mathrm{H}_{2}\right.$, $\mathrm{CH}_{3} \mathrm{C}_{4} \mathrm{H}$, and $\mathrm{HC}_{5} \mathrm{~N}$ ) with $\mathrm{He}$.

It is possible to extend MQCT to the case of two coupled rotors, which would permit inelastic scattering calculations of molecule + molecule collisions. Such developments are in progress. The relevant applications will include excitation/quenching of complex organic molecules and linear carbon chains by $\mathrm{H}_{2}$ and also collisions between two water molecules (including their isotopomers) and several other triatomic + triatomic systems of astrophysical relevance, which is way too complicated for the full-quantum treatment. Our estimates suggest that calculations for all of these processes are affordable within the framework of MQCT.

Although less relevant to astrophysics, another useful extension of MQCT is toward the high pressure regime, where the multiple collisions of a molecule with bath gas atoms/molecules cannot be treated independently, similar to the falloff regime of recombination kinetics where the three-body collisions are important. The timedependent formulation of MQCT should allow simulations in which the molecule would interact successively or simultaneously with two or

Journal of Physical Chemistry Letters, Vol 6, No. 10 (May 21, 2015): pg. 1854-1858. DOI. This article is (C) American Chemical Society and permission has been granted for this version to appear in e-Publications@Marquette. American Chemical Society does not grant permission for this article to be further copied/distributed or hosted elsewhere without the express permission from American Chemical Society. 
more quenchers along one trajectory. Such theory would have numerous applications in the high-pressure combustion.

The authors declare no competing financial interest.

\section{Acknowledgment}

K. Szalewicz is gratefully acknowledged for sharing his PES for interaction of methyl formate with He atom. L. Wiesenfeld is acknowledged for stimulating discussions. Financial support: this research was partially supported by NSF, through the grant AGS1252486. This research used resources of the National Energy Research Scientific Computing Center, which is supported by the Office of Science of the U.S. Department of Energy under contract no. DEAC02-5CH11231.

\section{References}

${ }^{1}$ Roueff, E.; Lique, F.Molecular Excitation in the Interstellar Medium: Recent Advances in Collisional, Radiative, and Chemical Processes Chem. Rev. 2013, 113, 8906- 8938

2 Dubernet, M.-L.; Alexander, M. H.; Ba, Y. A.; Balakrishnan, N.; Balana, C.; Ceccarelli, C.; Cernicharo, J.; Daniel, F.; Dayou, F.; Doronin, M.BASECOL2012: a Collisional Database Repository and Web Service within the Virtual Atomic and Molecular Data Centre (VAMDC) Astron. Astrophys. 2013, A50, 553- 566

3 Tielens, A. G. The Physics and Chemistry of the Interstellar Medium; CUP: Cambridge, U.K., 2006.

${ }^{4}$ Caselli, P.; Ceccarelli, C.Our Astrochemical Heritage Astron. Astrophys. Rev. 2012, 20 ( 56) 1- 68

${ }^{5}$ Sakai, N.; Yamamoto, S.Warm Carbon-Chain Chemistry Chem. Rev. 2013, $113,8981-9015$

${ }^{6}$ Henning, T.; Semenov, D.Chemistry in Protoplanetary Disks Chem. Rev. 2013, 113, 9016- 9042

7 Arthurs, A. M.; Dalgarno, A.The Theory of Scattering by a Rigid Rotator Proc. R. Soc. 1960, A256, 540- 551

${ }^{8}$ Green, S.Rotational Excitation of Symmetric Top Molecules by Collisions with Atoms: Close Coupling, Coupled States, And Effective Potential Calculations for $\mathrm{NH}_{3}-\mathrm{He}$ J. Chem. Phys. 1976, 64, 3463- 3473

${ }^{9}$ Wernli, M.; Wiesenfeld, L.; Faure, A.; Valiron, P.Rotational Excitation of $\mathrm{HC}_{3} \mathrm{~N}$ by $\mathrm{H}_{2}$ and $\mathrm{He}$ at Low Temperatures Astron. Astrophys. 2007, 464, 1147- 1154

Journal of Physical Chemistry Letters, Vol 6, No. 10 (May 21, 2015): pg. 1854-1858. DOI. This article is @ American Chemical Society and permission has been granted for this version to appear in e-Publications@Marquette. American Chemical Society does not grant permission for this article to be further copied/distributed or hosted elsewhere without the express permission from American Chemical Society. 
NOT THE PUBLISHED VERSION; this is the author's final, peer-reviewed manuscript. The published version may be accessed by following the link in the citation at the bottom of the page.

${ }^{10}$ Daniel, F.; Dubernet, M.-L.; Grosjean, A.Rotational Excitation of 45 Levels of ortho/para- $\mathrm{H}_{2} \mathrm{O}$ by Excited ortho/para- $\mathrm{H}_{2}$ from 5 to $1500 \mathrm{~K}$ : Stateto-State, Effective, And Thermalized Rate Coefficients Astron.

Astrophys. 2011, A76, 536- 546

${ }^{11}$ Faure, A.; Szalewicz, K.; Wiesenfeld, L.Potential Energy Surface and Rotational Cross Sections for Methyl Formate Colliding with Helium $J$. Chem. Phys. 2011, 135, 024301-024301(10)

12 Rabli, D.; Flower, D. R.The Rotational Excitation of Methanol by Molecular Hydrogen Mon. Not. R. Astron. Soc. 2010, 406, 95

13 Troscompt, N.; Faure, A.; Wiesenfeld, L.; Ceccarelli, C.; Valiron, P.Rotational Excitation of Formaldehyde by Hydrogen Molecules: ortho- $\mathrm{H}_{2} \mathrm{CO}$ at Low Temperature Astron. Astrophys. 2009, 493, 687696

${ }^{14}$ Semenov, A.; Babikov, D.Equivalence of the Ehrenfest Theorem and the Fluid-Rotor Model for Mixed Quantum/Classical Theory of Collisional Energy Transfer J. Chem. Phys. 2013, 138, 164110-164110(10)

15 Semenov, A.; Babikov, D.Mixed Quantum/Classical Theory of Rotationally and Vibrationally Inelastic Scattering in Space-Fixed and Body-Fixed Reference Frames J. Chem. Phys. 2013, 139, 174108-174108(15)

${ }^{16}$ Semenov, A.; Ivanov, M. V.; Babikov, D.Ro-Vibrational Quenching of CO (v $=1$ ) by He Impact in a Broad Range of Temperatures: A Benchmark Study Using Mixed Quantum/Classical Inelastic Scattering theory J. Chem. Phys. 2013, 139, 074306-074306(12)

17 Semenov, A.; Babikov, D.Accurate Calculations of Rotationally Inelastic Scattering Cross Sections Using Mixed Quantum/Classical Theory J. Phys. Chem. Lett. 2014, 5, 275- 278

18 Semenov, A.; Babikov, D. Mixed Quantum/Classical Calculations of Total and Differential Elastic and Rotationally Inelastic Scattering Cross Sections for Light and Heavy Reduced Masses in a Broad Range of Collision Energies J. Chem. Phys. 2014, 140, 044306-044306(13)

19 Semenov, A.; Dubernet, M.-L.; Babikov, D.Mixed Quantum/Classical Theory for Inelastic Scattering of Asymmetric-Top-Rotor + Atom in the BodyFixed Reference Frame and Application to the $\mathrm{H}_{2} \mathrm{O}+\mathrm{He}$ System $\mathrm{J}$. Chem. Phys. 2014, 141, 114304-114304(8)

20 Levine, R. D. Quantum Mechanics of Molecular Rate Processes; Oxford University Press: Oxford, U.K., 1969.

${ }^{21}$ Prezhdo, O. V.Quantized Hamilton Dynamics Theor. Chem. Acc. 2006, 116, 206- 218

22 Prezhdo, O. V.; Pereverzev, Y. V.Quantized Hamilton Dynamics J. Chem. Phys. 2000, 113, 6557

${ }^{23}$ Booksby, C.; Prezhdo, O. V. Quantized Mean-Field Approximation Chem. Phys. Lett. 2001, 346, 463- 469

Journal of Physical Chemistry Letters, Vol 6, No. 10 (May 21, 2015): pg. 1854-1858. DOI. This article is (c) American Chemical Society and permission has been granted for this version to appear in e-Publications@Marquette. American Chemical Society does not grant permission for this article to be further copied/distributed or hosted elsewhere without the express permission from American Chemical Society. 
NOT THE PUBLISHED VERSION; this is the author's final, peer-reviewed manuscript. The published version may be accessed by following the link in the citation at the bottom of the page.

24 Billing, G. D.the Semiclassical Treatment of Molecular Roto-Vibrational Energy Transfer Comput. Phys. Rep. 1984, 1, 237- 296

25 Billing, G. D. The Quantum Classical Theory; Oxford University Press: Oxford, U.K., 2002.

26 McGuire, P.; Kouri, D. J.Quantum Mechanical Close Coupling Approach to Molecular Collisions. jz -Conserving Coupled States Approximation J. Chem. Phys. 1974, 60, 2488- 2499

27 Alexander, M. H.; Manolopoulos, D. E.; Werner, H. J.; Vohralik, P. F.; Lemoine, D.; Corey, G.; Gordon, R.; Johnson, B.; Orlikowski, T.; Berning, A.; Hibridon; University of Maryland: College Park, MD, 2011.

${ }^{28}$ Hutson, J. M.; Green, S. MOLSCAT Computer Code, version 14; 1994. Distributed by Collaborative Computational Project No. 6 of the Engineering and Physical Sciences Research Council, Swindon, U.K.

\section{Supporting Information}

Details of our theory. The Supporting Information is available free of charge on the ACS Publications website at DOI: 10.1021/acs.jpclett.5b00496.

\section{Mixed Quantum/Classical Approach for Description of Molecular Collisions in Astrophysical Environments}




\section{Mixed Quantum/Classical Approach for Description of Molecular Collisions in Astrophysical}

\section{Environments}

Alexander Semenov and Dmitri Babikov.

Chemistry Department, Wehr Chemistry Building, Marquette University,

Milwaukee, Wisconsin 53201-1881, USA

AUTHOR INFORMATION

Corresponding Author: Dmitri Babikov, Ph.D., Professor;

Phone: (414) 288-3538; e-mail address: dmitri.babikov@mu.edu.

\section{SUPPLEMENTARY MATERIALS}

The MQCT for a general molecule + atom system

In MQCT for a molecule + atom system the time-dependent rotational wave function of the molecule $\psi\left(\alpha^{\prime}, \beta^{\prime}, \gamma^{\prime}, t\right)$ is expanded over basis set of rotational eigenstates $\Psi_{m^{\prime} n}\left(\alpha^{\prime}, \beta^{\prime}, \gamma^{\prime}\right)$ using the time-dependent coefficients $a_{m^{\prime} n}(t)$ as follows:

$$
\psi\left(\alpha^{\prime}, \beta^{\prime}, \gamma^{\prime}, t\right)=\sum_{m^{\prime} n} a_{m^{\prime} n}(t) \Psi_{m^{\prime} n}\left(\alpha^{\prime}, \beta^{\prime}, \gamma^{\prime}\right) \exp \left\{-i E_{n} t / \hbar\right\}
$$

Primed Euler angles $\left(\alpha^{\prime}, \beta^{\prime}, \gamma^{\prime}\right)$ define position of the molecule in the BF reference frame, where axis $z$ is aligned along the molecule-atom direction. Index $n$ is a composite index that labels 
states and its meaning depends on the system. For the general case of an asymmetric top rotor, as methyl formate, we should set $n \equiv\left\{j, k_{a}, k_{c}\right\}$ and $\Psi_{m^{\prime} n} \equiv \Psi_{m^{\prime} k_{a} k_{c}}^{j}$. The energy $E_{n}$ of an eigenstate depends on $n$ only, and does not depend on $m^{\prime}$, which is projection of angular momentum $j$ of the molecule onto $z$-axis in the BF reference frame. Note that although we neglect the vibrational excitation and focus on rotational transitions only, inclusion of vibrational eigenstates (e.g., torsion of the methyl group) into the basis set expansion is rather straightforward.

Starting with expansion (1) and following the derivations outlined in Ref. [17], one can derive the general MQCT equations for time-evolution of probability amplitudes $a_{m^{\prime} n}(t)$ (quantum part of the system) and for time-evolution of the classically treated degrees of freedom in the problem $\{R, \Phi, \Theta\}$. These coordinates define the molecule-atom separation and the direction of the atom-molecule axis (which is the $\mathrm{BF} z$-axis) with respect to the laboratory reference frame. Here, we present just the final equations, adopted to the case when the initial rotational wave function $\psi\left(\alpha^{\prime}, \beta^{\prime}, \gamma^{\prime}, t\right)$ is a rotational eigenfunction, rather than a general rotational wave packet. In this special case the rotational wave function possesses cylindrical symmetry and the classical trajectory of motion $\{R(t), \Phi(t), \Theta(t)\}$ is restricted to one plane. It is the best to choose this plane to be the equatorial plane $\Theta=\pi / 2$, which greatly simplifies both classical and quantum equations of motion. In this case the time-dependent Schrodinger equation for atom-molecule scattering is reduced to the following system of coupled equations for probability amplitudes:

$$
i \hbar \frac{\partial a_{m^{\prime} n^{\prime}}}{\partial t}=\sum_{n^{\prime \prime}} a_{m^{\prime} n^{\prime \prime}} \exp \left\{i\left(E_{n^{\prime \prime}}-E_{n^{\prime}}\right) t / \hbar\right\} M_{n^{\prime}}^{n^{\prime \prime}}+\hbar \sum_{m^{\prime \prime}} a_{m^{\prime \prime} n^{\prime}} V_{m^{\prime}}^{m^{\prime \prime}} \dot{\Phi}
$$

Here the matrix $\mathbf{V}$ describes transitions between $m^{\prime}$-components of $j$ in the BF reverence frame. It is computed analytically for every $j$ as follows:

$$
V_{m^{\prime}}^{m^{\prime \prime}}=\frac{1}{2}\left[\sqrt{j(j+1)-m^{\prime \prime}\left(m^{\prime \prime}-1\right)} \delta_{m^{\prime}, m^{\prime \prime}-1}+\sqrt{j(j+1)-m^{\prime \prime}\left(m^{\prime \prime}+1\right)} \delta_{m^{\prime}, m^{\prime \prime}+1}\right] .
$$

The last term in Eq. (2) occurs in the BF formalism only [17], not in the SF formalism, and the coupled-states approximation is obtained readily by neglecting this term [18-19]. Note that matrix $\mathbf{V}$ is time-independent (should be computed only once) and is analytic. It doesn't involve 
any interaction potential. In contrast, matrix $\mathbf{M}$ in Eq. (2) describes transitions between states $n$, and is computed for every $m^{\prime}$-component of $j$ as follows:

$$
M_{n^{\prime}}^{n^{\prime \prime}}(R)=\left\langle\Psi_{m^{\prime} n^{\prime \prime}}\left(\alpha^{\prime}, \beta^{\prime}, \gamma^{\prime}\right)\left|V\left(R, \alpha^{\prime}, \beta^{\prime}, \gamma^{\prime}\right)\right| \Psi_{m^{\prime} n^{\prime}}\left(\alpha^{\prime}, \beta^{\prime}, \gamma^{\prime}\right)\right\rangle
$$

This is a potential coupling matrix. Its elements include the interaction potential and should be computed numerically. Elements of $\mathbf{M}$ are real and depend on $R$ only.

In the case of a general asymmetric-top rotor the rotational wave function is expressed as follows:

$$
\Psi_{m^{\prime} k_{a} k_{c}}^{j}\left(\alpha^{\prime}, \beta^{\prime}, \gamma^{\prime}\right)=\sqrt{\frac{2 j+1}{8 \pi^{2}}} \sum_{k=-j}^{j} b_{j k_{a} k_{c}}^{k} D_{m^{\prime} k}^{j}\left(\alpha^{\prime}, \beta^{\prime}, \gamma^{\prime}\right),
$$

where the coefficients $b_{j k_{a} k_{c}}^{k}$ are obtained by numerical diagonalization of the rotational Hamiltonian of the molecule. A standard approach in the inelastic scattering calculations is to expand the molecule-atom interaction potential over basis set of spherical harmonics, which permits to express elements of the state-to-state transition matrix in a convenient analytic form. To accommodate this scheme we derived the required expressions for elements of $M_{n^{\prime}}^{n^{\prime \prime}}(R)$ for a general case of an asymmetric-top rotor + atom system [19]. Although this approach works well for small and simpler molecules it is known that for larger and more complicated molecules, such as methyl formate, the analytic potential expansion converges poorly and requires truncation techniques that are hard to justify $[9,11]$. To avoid these problems we followed a different approach and computed the elements of the transition matrix by numerical integration in Eq. (4). It should be emphasized that in a molecule + atom system the interaction potential does not depend on depend $\alpha^{\prime}$, so, $V=V\left(R, \beta^{\prime}, \gamma^{\prime}\right)$. Furthermore, the dependence of rotational wave function of Eq. (5) on Euler angles is given explicitly by:

$$
\Psi_{m^{\prime} k_{a} k_{c}}^{j}\left(\alpha^{\prime}, \beta^{\prime}, \gamma^{\prime}\right)=\sqrt{\frac{2 j+1}{8 \pi^{2}}} \sum_{k=-j}^{j} b_{j k_{a} k_{c}}^{k} \exp \left(i m^{\prime} \alpha^{\prime}\right) d_{m^{\prime} k}^{j}\left(\beta^{\prime}\right) \exp \left(i k \gamma^{\prime}\right) .
$$

This means that the phase factor $\exp \left(\mathrm{im}^{\prime} \alpha^{\prime}\right)$ cancels out analytically in the integration of Eq. (4) and the potential coupling matrix $M_{j^{\prime} k_{a}^{\prime} k_{c}^{\prime}}^{j^{\prime \prime} k_{c}^{\prime \prime}}(R)$ is diagonal with respect to $m^{\prime}$. Numerical 
integration over $\beta^{\prime}$ and $\gamma^{\prime}$ was carried out using a Legendre-Gauss quadrature on a twodimensional $50 \times 50$ grid, determined by convergence studies.

Differential equations for classical degrees of freedom $\{R(t), \Phi(t)\}$ also include matrixes $\mathbf{M}$ and $\mathbf{V}$, as a commutator [19]:

$$
\begin{aligned}
& \dot{R}=\frac{P_{R}}{\mu} \\
& \dot{\Phi}=\frac{P_{\Phi}}{\mu R^{2}} \\
& \dot{P}_{R}=-\sum_{m^{\prime \prime} n^{\prime \prime}} \sum_{m^{\prime} n^{\prime}} a_{m^{\prime \prime} n^{\prime \prime}}^{*} a_{m^{\prime} n^{\prime}} \exp \left\{i\left(E_{n^{\prime \prime}}-E_{n^{\prime}}\right) t / \hbar\right\} \frac{\partial M_{n^{\prime}}^{n^{\prime \prime}}(R)}{\partial R}+\frac{P_{\Phi}^{2}}{\mu R^{3}} \\
& \dot{P}_{\Phi}=-i \sum_{m^{\prime \prime} n^{\prime \prime}} \sum_{m^{\prime} n^{\prime}} a_{m^{\prime \prime} n^{\prime \prime}}^{*} a_{m^{\prime} n^{\prime}} \exp \left\{i\left(E_{n^{\prime \prime}}-E_{n^{\prime}}\right) t / \hbar\right\}[\mathbf{M}, \mathbf{V}]_{m^{\prime} n^{\prime}}^{m^{\prime \prime}} .
\end{aligned}
$$

As we showed in Ref. [17], expressions in the right-hand sides of Eqs. (9-10) are real-valued, leading to the real-valued classical momenta and their time-derivatives. Such equations can be easily propagated numerically, just as classical trajectories of motion.

Sampling of the classical initial conditions, and the final analysis of transition amplitudes $a_{m^{\prime} n^{\prime}}(t=+\infty)$ to compute cross sections, are closely interconnected issues. Absolute value $P$ of the initial momentum $\mathbf{P}$ is determined by incident energy of collision $E=P^{2} / 2 \mu$, while various possible directions of $\mathbf{P}$ in space correspond to different values of $\ell=|\mathbf{I}|$ and $J=|\mathbf{J}|$, where $\mathbf{l}$ is the orbital angular momentum, and $\mathbf{J}=\mathbf{l}+\mathbf{j}$ is the total angular momentum. The case of $j=0$ considered in this paper is particularly simple: since $J=\ell$ the integration is one dimensional and can be carried out using a regular equidistant grid (the general case of $j>0$ is implemented most efficiently using a two-dimensional Monte-Carlo sampling over $J$ and $\ell$, as discussed in Ref. [16]). Namely, we set up a grid of $N$ sample points between $J=0$ and $J_{\max }$, and for every point we define the value of initial classical momentum $P_{\Phi}=\hbar \sqrt{\ell(\ell+1)}$ , to use in Eq. (8-9). The value of $\ell=J$ is closely related to the collision impact parameter $b$ through $\ell(\ell+1)=k^{2} b^{2}$ and $\mathbf{k}=\mathbf{P} / \hbar$. The value of $P_{R}$, to use in Eq. (7), is computed for each 
point from $\mathbf{P}^{2}=P_{R}^{2}+P_{\Phi}^{2} / R^{2}$. Thus, $N$ classical trajectories are initiated (labeled by $i$ ) and the inelastic scattering cross section is determined numerically as:

$$
\sigma_{n^{\prime} m^{\prime} \rightarrow n^{\prime \prime} m^{\prime \prime}}=\frac{\pi}{k^{2}} \frac{J_{\max }}{N} \sum_{i}\left(2 J^{(i)}+1\right) p_{n^{\prime \prime} m^{\prime \prime}}^{(i)}
$$

where $p_{n^{\prime \prime} m^{\prime \prime}}=\left|a_{n^{\prime \prime} m^{\prime \prime}}(t=+\infty)\right|^{2}$ is transition probability for a given trajectory. We want to emphasize that MQCT trajectories are not binned into any "boxes" at the final moment of time. Each MQCT trajectory, started in a given initial state $n^{\prime} m^{\prime}$, makes contribution to every final state $n^{\prime \prime} m^{\prime \prime}$, according to the values of $a_{n^{\prime \prime} m^{\prime \prime}}(t=+\infty)$. This feature results in favorable convergence properties of the method and requires only a moderate number of MQCT trajectories. The sampling of MQCT trajectories over $J$ and $\ell$ is similar to sampling of purely classical trajectories over impact parameter, since $J_{\max }=k \hbar b_{\max }$. In this work, the maximum value of impact parameter determined by convergence studies was $b_{\max }=15 a_{0}\left(J_{\max }=64\right)$, sufficient even at lower collision energies, and more than sufficient at higher collision energies. The initial molecule-atom separation $R$ was $16 a_{0}$. The total number of classical trajectories was around $N=200$ at each scattering energy, providing convergence of cross section with respect to this parameter on order of $1-2 \%$. This number of trajectories is not particularly large because we only have to sample over $J=\ell$, as explained above, and because the dependence of transition probabilities on impact parameter is rather simple, as one can see from Fig. 1. In fact, the number of trajectories can be reduced even further, if more intelligent integration technique is employed. The number of channels needed for MQCT calculations was also checked by convergence studies. For example, for methyl formate collided with He at energy near $150 \mathrm{~cm}^{-1}$ we included 146 closed channels, in addition to 488 open (energetically accessible) channels.

\section{Coupled-states approximation within MQCT framework}

An approximate version of MQCT is easily obtained if transitions between $m$-states are neglected, by zeroing out matrix $\mathbf{V}$ of Eq. (3). This leads to a simplified equation for evolution of probability amplitudes, instead of (2): 


$$
i \hbar \frac{\partial a_{m^{\prime} n^{\prime}}}{\partial t}=\sum_{n^{\prime \prime}} a_{m^{\prime} n^{\prime \prime}} \exp \left\{i\left(E_{n^{\prime \prime}}-E_{n^{\prime}}\right) t / \hbar\right\} M_{n^{\prime}}^{n^{\prime \prime}}
$$

Note that this equation does not include any classical variables. It is propagate independently for every value of the projection quantum number $m$.

The classical equations of motion also simplify. Instead of (7-10) we obtain:

$$
\begin{aligned}
& \dot{R}=\frac{P_{R}}{\mu}, \\
& \dot{P}_{R}=-\sum_{m^{\prime \prime} n^{\prime \prime}} \sum_{m^{\prime} n^{\prime}} a_{m^{\prime \prime} n^{\prime \prime}}^{*} a_{m^{\prime} n^{\prime}} \exp \left\{i\left(E_{n^{\prime \prime}}-E_{n^{\prime}}\right) t / \hbar\right\} \frac{\partial M_{n^{\prime}}^{n^{\prime \prime}}(R)}{\partial R}+\frac{P_{\Phi}^{2}}{\mu R^{3}} .
\end{aligned}
$$

As you see, equations for the azimuthal angle $\Phi$ don't have to be propagated at all. Namely, the value of $\Phi(t)$ becomes irrelevant, while the value of $P_{\Phi}$, determined by the initial sampling, remains constant along the CS-MQCT trajectory. Basically, only the radial degree of freedom is retained. This form of MQCT is very similar to the method of Billing [21,22], except that he restricted trajectories to the polar plane $\Phi=0$. We use the equatorial plane $\Theta=\pi / 2$ instead, in order to avoiding singularity at the "North Pole" in the fully-coupled CC-MQCT equations.

Equations (12-14) are much faster to integrate. In the case of methyl formate + He the speed up of CS-MQCT calculations is by a factor of 20, compared to the fully coupled CCMQCT of equations (2) and (7-10). But, as it is demonstrated in this paper and in our other recent work [17], the accuracy often suffers. 